\title{
Spin-up of the hyperon-softened accreting neutron stars
}

\author{
J. L. Zdunik, P. Haensel, and M. Bejger
}

\author{
N. Copernicus Astronomical Center, Polish Academy of Sciences, Bartycka 18, 00-716 Warszawa, Poland \\ e-mail: [haensel;jlz; bejger]@camk.edu.pl
}

Received 7 February 2005 / Accepted 25 April 2005

\begin{abstract}
We study the spin-up of the accreting neutron stars with a realistic hyperon-softened equation of state. Using precise 2D calculations we study the evolutionary tracks of accreting neutron stars in the angular-momentum - frequency planes. In contrast to the case of spinning-down solitary radio-pulsars, where a strong back-bending behavior has been observed, we do not see back-bending phenomena in the accretion-powered spinning-up case. We conclude that in the case of accretion-driven spin-up the back-bending is strongly suppressed by the mass-increase effect accompanying the angular-momentum increase.
\end{abstract}

Key words. dense matter - equation of state - stars: neutron - stars: binaries: general

\section{Introduction}

Some theories of dense matter predict a phase transition to a new "exotic" state of matter at densities higher than the nuclear saturation density. A phase transition softens the equation of state (EOS). It has in turn been suggested that it leads to characteristic astrophysical signatures, for example, very large values of the braking index of spinning-down radio-pulsars, as well as period-clustering of spinning-up neutron stars in lowmass X-ray binaries (Glendenning et al. 1997; Glendenning \& Weber 2001a,b; Poghosyan et al. 2001; Glendenning \& Weber 2002). Detection of any of these specific features might serve as a clue to a deeper understanding of the EOS of matter at supranuclear densities.

This work is a consecutive step in our studies of the relation between the realistic EOS of dense matter, dynamics of neutron stars, their evolutionary tracks, and observational signatures. As in our previous article (Zdunik et al. 2004, hereafter referred to as Paper I) we would like to focus on the phenomenon of back-bending, which is a very characteristic consequence of the softening of the EOS on the spin evolution of a neutron star.

In Paper I we showed that the back-bending in spinningdown solitary pulsars is not necessarily evidence of an "exotic" phase of dense matter. Specifically, it was demonstrated that the occurrence of back-bending can also be caused by hyperons, particles whose presence in dense matter was predicted over forty years ago (Cameron 1959). We also demonstrated the crucial importance of precision in numerical calculations and of checking the stability of quasi-stationary rotating configurations in the back-bending region.

In the present paper we describe numerical simulations of the mass and angular momentum transfer onto a neutron star with the EOSs involving hyperons. In particular, we consider

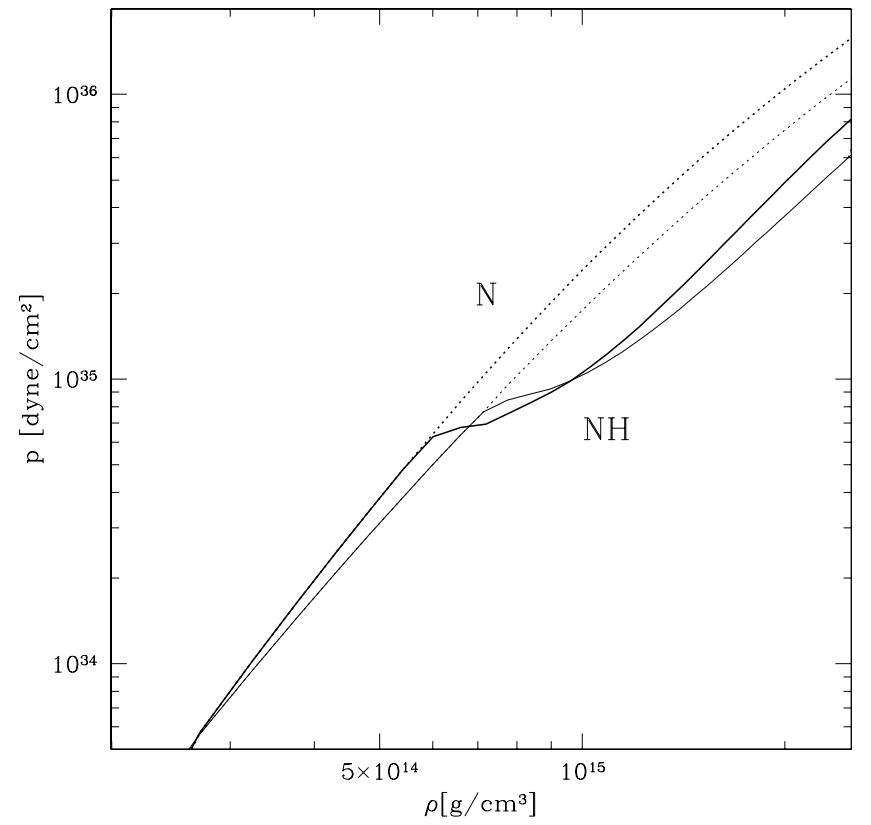

Fig. 1. Solid lines NH: pressure $p$ vs. mass density $\rho$ for the EoS1 $N \Lambda \Xi$ and EoS2 $N \Lambda \Xi$ of Balberg et al. (1999), calculated by Balberg \& Gal (1997) (N1H1 and N2H1 EOS in the notation of Paper I). Dotted lines were obtained by suppressing the hyperons, i.e., by assuming purely nucleon dense matter. The visible softening around $\rho \sim 7 \times 10^{14}$ for the $\mathrm{NH}$ models results from the appearance of hyperons in dense matter.

the equation EoS2 N $\triangle \Xi$ (N2H1 in notation of Paper I) of Balberg et al. (1999), which according to Paper I, revealed the strongest back-bending effect in spinning-down neutron stars. We present results obtained for two EOSs softened by the appearance of hyperons (out of four EOSs calculated by Balberg et al. 1999) which correspond to two different models of purely 
nucleon matter. One of them, N1, has a standard incompressibility of nuclear matter $K=240 \mathrm{MeV}$. The second model, N2, corresponds to a rather stiff nuclear matter with $K=320 \mathrm{MeV}$. Consequently, N2 gives an equation of state that is noncausal at very high density. However, it should be stressed that all stellar configurations presented in our paper have central density well below this non-causality threshold.

Our model is described in Sect. 2. Results of numerical calculations are collected in Sect. 3, while Sect. 4 contains discussion and concluding remarks.

\section{Astrophysical model}

We assume that the evolution of an accreting neutron star can be represented as a sequence of stationary rotating configurations of increasing baryon mass. In what follows we assume that matter is accreted from an accretion disc in which elements of matter move on Keplerian orbits, moving slowly inward due to the viscous angular momentum outward transport. The magnetic field is assumed to be sufficiently weak so as not to affect the disc dynamics. However, it may still play an important role in the angular momentum transport. The inner part of the disc is assumed to be geometrically thin, so that the approximation of accretion of matter in the equatorial plane is good.

Two radii of co-axial circles in the equatorial plane play a crucial role in our calculation. The first one, denoted $R_{\text {eq }}$, is the radial coordinate of the neutron star surface at the equator. The second important radial coordinate is the radius of the marginally stable orbit of a particle in the space-time around and within a rotating neutron star, $r_{\mathrm{ms}}$. Orbital motion of particles on the orbits with $r>r_{\mathrm{ms}}$ is stable while that of particles on circular orbits with $r<r_{\mathrm{ms}}$ is unstable. Two accretion regimes are considered, as explained below.

The first scenario corresponds to $r_{\mathrm{ms}}>R_{\mathrm{eq}}$. We then calculate the spin-up of an accreting neutron star according to the prescription given by Zdunik et al. (2002). Matter is accreted from the marginally stable orbit (MSO) with $r=r_{\mathrm{ms}}$, which determines the inner edge of the accretion disc. A particle falls from the MSO onto the neutron star surface after losing an infinitesimal amount of its angular momentum due to viscous processes. We denote the specific angular momentum per unit baryon mass by $l_{\mathrm{ms}}$ at the MSO. A more detailed discussion concerning determination of MSO and $l_{\mathrm{ms}}$ in the framework of General Relativity can be found in Zdunik et al. (2002).

In the second case $r_{\mathrm{ms}}<R_{\mathrm{eq}}$, and MSO is located inside the star. In this case we assume that the inner edge of the accretion disc is connected to stellar surface via a thin transition layer, where viscous processes transport the angular momentum to the neutron star.

In both cases we define the innermost stable circular orbit (ISCO), from which the angular momentum and baryon mass is transferred to a neutron star. The ISCO determines the inner edge of the accretion disc. We use the approximation $r_{\mathrm{ISCO}}=r_{\mathrm{ms}}$ for $r_{\mathrm{ms}}>R_{\mathrm{eq}}$ and $r_{\mathrm{ISCO}}=R_{\mathrm{eq}}$ for $r_{\mathrm{ms}}<R_{\mathrm{eq}}$.

The value of specific angular momentum per unit baryon mass of a particle orbiting the neutron star at the ISCO, $l_{\mathrm{IS}}$, can be calculated by solving exact equations of the orbital motion of a particle in the space-time produced by a rotating neutron star, given in Appendix A of Zdunik et al. (2002).

Accretion of an infinitesimal amount of baryon mass $\mathrm{d} M_{\mathrm{B}}$ onto a rotating neutron star is assumed to lead to a new quasi stationary rigidly rotating configuration of mass $M_{\mathrm{B}}+\mathrm{d} M_{\mathrm{B}}$ and angular momentum $J+\mathrm{d} J$, with

$\mathrm{d} J=x_{l} l_{\mathrm{IS}} \mathrm{d} M_{\mathrm{B}}$,

where $x_{l}$ denotes the fraction of the angular momentum of the element of matter transferred to the star. The remaining fraction $1-x_{l}$ might be lost via radiation or other dissipative processes. In the case of $r_{\mathrm{ms}}>R_{\mathrm{eq}}$, these processes are acting on the MSO and during the fall of an element of matter through the dilute but magnetized plasma and the photon radiation emitted from stellar surface, which fills the gap between the MSO and stellar surface (Agol \& Krolik 2000; Afshordi \& Paczynski 2003). In the case of $r_{\mathrm{ISCO}}=R_{\mathrm{eq}}$, the dissipation and the angular momentum loss take place within the boundary layer, through which the accretion disk joins the stellar surface (see, e.g., Popham \& Sunyaev 2001, and references therein).

\section{Calculations of evolutionary tracks}

We assume that the evolution of an accreting neutron star can be represented as a sequence of stationary rotating $2 \mathrm{D}$ configurations. The numerical method used for the calculation of configurations belonging to this family was described in Sect. 3 of Paper I. Here, we would like to stress the very high precision of our calculations; evaluation of the GRV2 and GRV3 virial error indicators (see Nozawa et al. 1998, and references therein) showed relative errors within $\simeq 10^{-5}$.

To study the effect of hyperons on the evolution of accreting neutron star, we compare the results for N1 and N1H1 EOSs, in the notation of Paper I, the models of Balberg \& Gal (1997). In Fig. 2 we present the evolutionary tracks in $n_{\mathrm{c}}-M$ plane, where $n_{\mathrm{c}}$ is the central baryon density and $M$ is gravitational mass of NS. Using this figure we can answer the question how accretion changes physical conditions in the center of a star. As we can see for $x_{l}=1$, the curves for $\mathrm{N}$ (nucleons only) stars are almost vertical. It means that accretion results in the increase of $f$ (and $J$ ), but the density in the center of the star remains essentially unchanged. In the case of NH EOS (with hyperons), at the first stage of accretion the density in the center increases, as well as the size of the hyperon core. However, the main effect of the mass and angular momentum transfer is spin-up and change of shape (deformation) of NS, accompanied by a decrease of $n_{\mathrm{c}}$. As in the case of strange stars (Zdunik et al. 2002), the effect strongly depends on the fraction, $x_{l}$, of the angular momentum transferred to the stars from the ISCO. For $x_{l}=0.5$, central density and the size of hyperon core increase during accretion, leading eventually to a collapse of the NS into a black hole; this is to be contrasted with the $x_{l}>0.7$ cases, where the termination point is the mass-shedding (Keplerian) limit. Considered examples show that, in order to build a rather large hyperonic core, a significant amount of angular momentum has to be lost in the course of accretion.

Another difference between the evolution of accreting NS with and without hyperon core is visualized in 


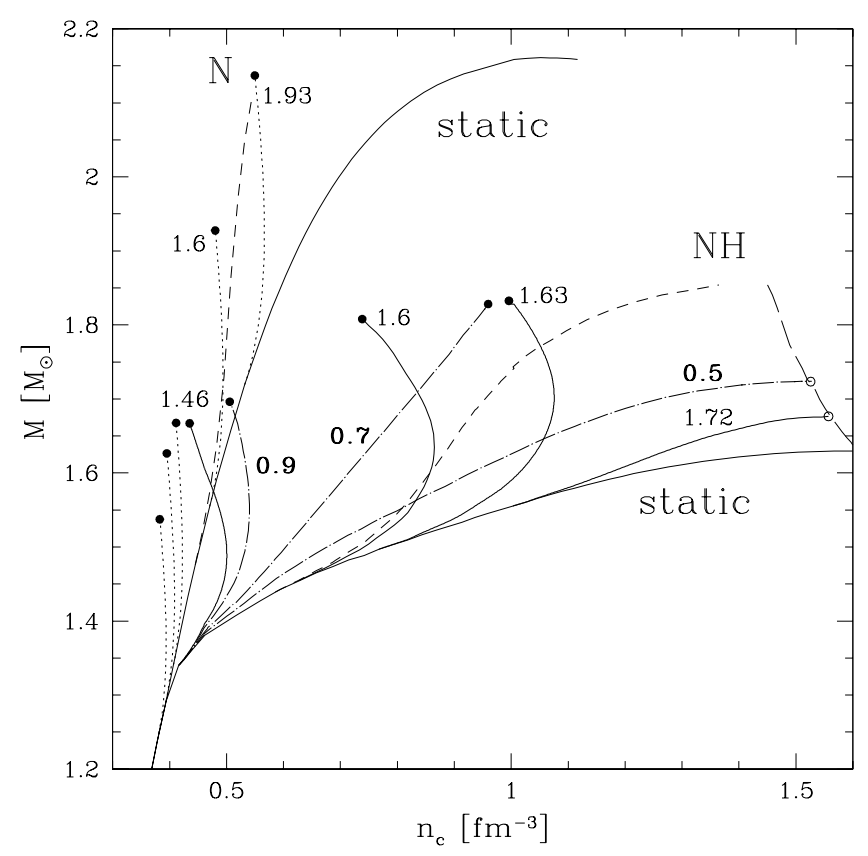

Fig. 2. Gravitational mass versus central baryon number density along several evolutionary tracks of accreting stars with hyperons (model N1H1) and without hyperons (model N1). Solid lines (model N1H1) and dotted lines (model N1) correspond to the typical case of all angular momentum of matter falling from the marginally stable orbit is transferred to the star $\left(x_{l}=1\right)$. Dash-dotted lines represent three cases for an $M_{0}=1.46 M_{\odot} \mathrm{N} 1 \mathrm{H} 1$ star with $x_{l}=0.9,0.7,0.5$, fractions indicated as boldface labels, from the left to the right. The long-dashed, nearly vertical line at maximum mass is defined by the onset of instability with respect to axisymmetric perturbations. Dashed lines correspond to the condition $R_{\mathrm{eq}}=r_{\mathrm{ms}}$. Termination points are marked by a filled circle for Keplerian configurations and by an open circle for the instability with respect to axisymmetric perturbation.

Fig. 3. For the $\mathrm{N} 1$ and $\mathrm{N} 1 \mathrm{H} 1$ EOSs, the evolution of spin frequency, as a function of angular momentum, depends on the mass of initial configuration in opposite ways. For $M_{\mathrm{B}, \text { in }}=1.46 M_{\odot}$, the evolution of these two EOSs does not differ significantly, and the curves almost coincide. The $\mathrm{N} 1$ stars with higher initial mass have larger $J$ for the same $f$. If a star contains a hyperon core, as for the N1H1 model, higher mass results in a lower $J$ for a given spin frequency. This effect can be explained by the specific dependence of the moment of inertia $I$ on the mass of the star. For slow rotation, the moment of inertia of the star is defined as a limit $I=\lim _{f \rightarrow 0} \mathrm{~d} J / \mathrm{d} f$. Let us recall that, except for $M$ close to maximum allowable mass for non-rotating NSs, $M_{\max }^{\text {stat }}$, the value of $I$ increases monotonically with $M$. However, close to $M_{\max }^{\text {stat }}$, a rapid decrease of $R$ with increasing $M$ dominates and makes $I$ decrease with increasing $M$. For the N1 model the initial configuration with $M_{\mathrm{B}} \sim 1.5-2 M_{\odot}$ is sufficiently far from the maximum mass and the increase with $M$ dominates. For the N1H1 model we are close to $M_{\max }$ and the decrease of $R$ with a relatively small change (increase) in $M$ dominates.

In Fig. 3 we also plot the sequence of spinning down configurations with hyperon core for isolated neutron star (fixed baryon mass $M_{\mathrm{B}}$ ). To make comparison easier, the baryon mass

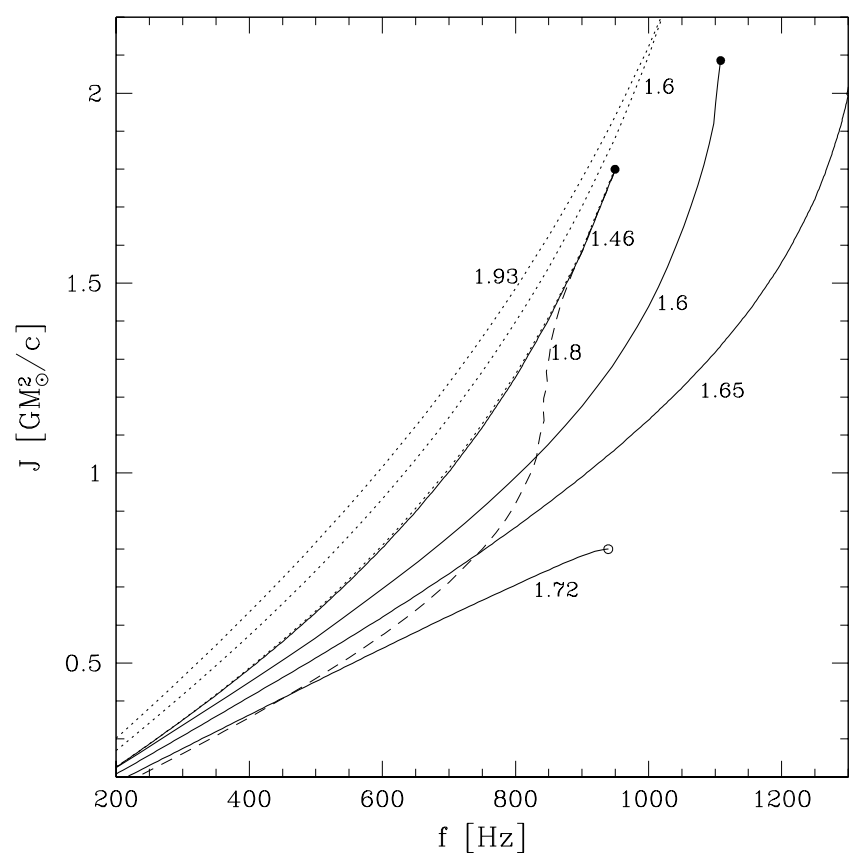

Fig. 3. Angular momentum as a function of spin frequency for accreting, spinning-up NS, with $x_{l}=1$ for EOS with (solid lines, N1H1 model) and without hyperons (dotted lines, N1 model). The label gives the baryon mass for the starting non-rotating configuration. The $J(f)$ track of an isolated NS with baryon mass of $1.8 M_{\odot}$ (thin dashed line) is also shown. To make comparison easier, the initial rotating state of spinning down isolated NS has been chosen to lie on the spin-up track of accreting NS with initial baryon mass of $1.46 M_{\odot}$. Termination points are marked by a filled circle for Keplerian configurations and by an open circle for the instability with respect to axisymmetric perturbation.

for this sequence $\left(1.8 M_{\odot}\right)$ is equal to the final mass of the accreting model with the initial mass $1.46 M_{\odot}$. The difference between the $J(f)$ dependence is evident; for isolated NS during slowing down the pulsar loses about half of its initial angular momentum without changing its rotation period much (in this case $\sim 800 \mathrm{~Hz}$ ). This effect is almost completely suppressed by accretion, as the derivative $\mathrm{d} J / \mathrm{d} f$ does not change significantly along the curve $M_{\mathrm{B}, \text { in }}=1.46 M_{\odot}$.

The same effect can be seen for the N2H1 EOS, for which we obtained the most pronounced back-bending behaviour for the isolated, spinning down neutron stars (Paper I). Results are presented in Figs. 4 and 5. In Fig. 4 we also plot the curve defined by the condition $R_{\mathrm{eq}}=r_{\mathrm{ms}}$, which is almost vertical and corresponds to the equatorial radius $13 \mathrm{~km}$. Marginally stable orbit exists to the left of this curve (i.e. equatorial radius smaller than $\sim 13 \mathrm{~km}$ ). The evolution of accreting NS, when $75 \%$ of the angular momentum is transferred to the star, can lead to the maximum accreted mass larger by more than $0.1 M_{\odot}$ than in the case of $x_{l}=1$ (typically the mass increase is $\sim 0.4 M_{\odot}$ ), before the star reaches the Keplerian limit (see Fig. 4). In Fig. 5 we use angular momentum as an argument (abscissa), because the change of angular momentum is directly connected with a time evolution, by Eq. (1), in the case of accretion and by the equation describing the energy and angular momentum loss due to the physical processes (e.g. magnetic dipole radiation) 


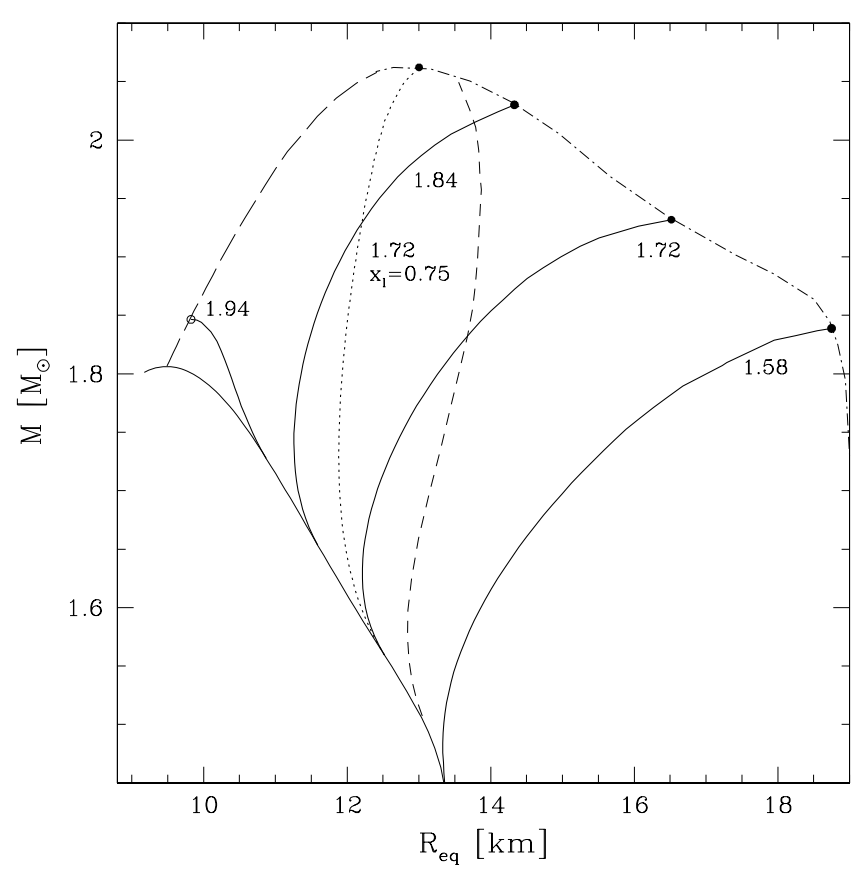

Fig. 4. Gravitational mass versus the (circumferential) equatorial radius, along several evolutionary tracks of accreting strange stars for $\mathrm{N} 2 \mathrm{H} 1$ model. The dotted line represents the model for which only $75 \%$ of angular momentum is transferred to the star. The longdashed line at maximum mass is defined by the onset of instability with respect to axisymmetric perturbations. The dash-dot line corresponds to the Keplerian (mass shedding) limit. The dashed line at $R_{\mathrm{eq}} \simeq 13 \mathrm{~km}$ corresponds to the condition $R_{\mathrm{eq}}=r_{\mathrm{ms}}$.

for isolated spinning down NS. The value of $l_{\text {IS }}$ does not change significantly during accretion period, so that the change of $J$ is roughly proportional to the accreted mass (i.e., to $\dot{M} \cdot t$ ).

For isolated neutron stars regions of back-bending exist (in Fig. 5 for $2.1 M_{\odot}$ ) and are denoted by thick dashed lines. The tiny thick-line region of spin-up of the star by angular momentum loss just before the onset of the axisymmetric instability (open circle), is a direct consequence of stability condition in GR (for other examples of this phenomenon, see Figs. 7-9 in Zdunik et al. 2004).

As one sees, there is no back-bending phenomenon for the accreting NSs, even in the most favorable case $x_{l}=1$, where the spinning-up of NS is the most efficient. This is due to a basic difference in the spin evolution of isolated and accreting NSs. For accreting NSs, increase in $J$ is necessarily accompanied with an increase in $M_{\mathrm{B}}$. This clearly weakens the impact of rotation on the stellar structure because of a simultaneous increase in $f$ and in the gravitational pull. Therefore, in this case spin-up by accretion cannot influence the structure of rotating NS so strongly as to enforce the back-bending behavior.

The total baryon mass accreted during the accretion spin-up epoch is obviously limited by the mass of the companion in the LMXB. This results in an upper bound on the final frequency reached at the end of accretion. It may happen, for example, that the spin-up tracks in Fig. 3 terminate well before the instability points.

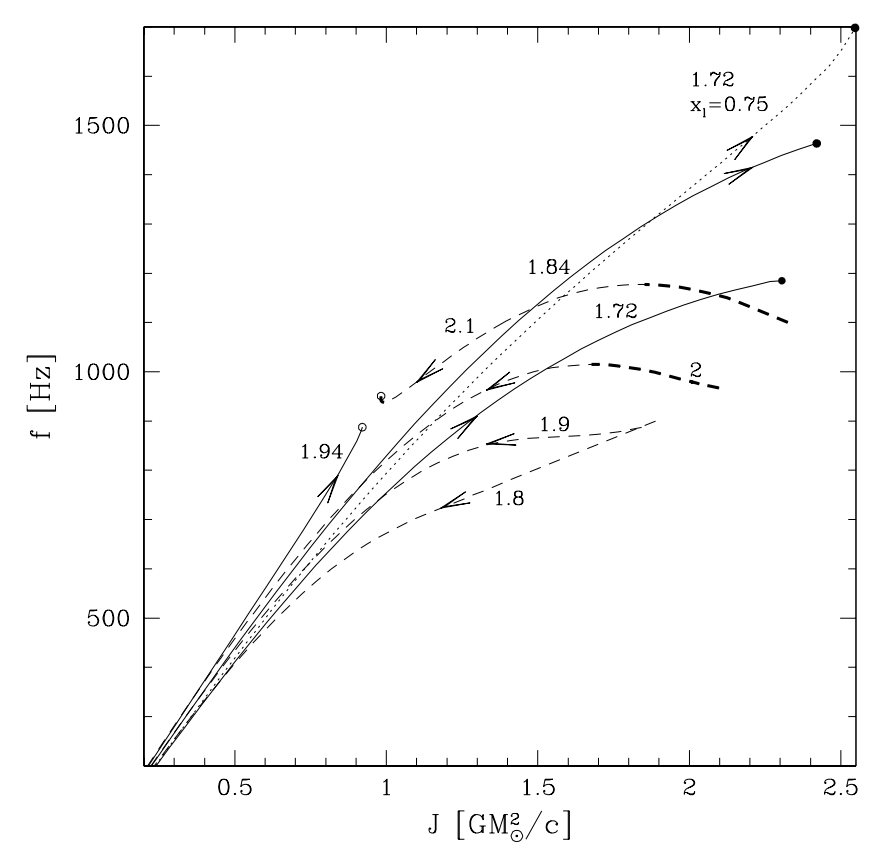

Fig. 5. Spin frequency as a function of angular momentum of the solitary, spinning-down pulsars (dashed lines) and accreting, spinning-up NS with $x_{l}=1$ (solid lines). In the case of dashed lines, the numbers denote their baryon mass $M_{\mathrm{B}}$ in the units of $M_{\odot}$, which stays constant along the evolutionary track. For accreting neutron-star spin-up tracks, the label gives the baryon mass for the starting non-rotating configuration. The arrows indicate the evolution of neutron-star configurations with time. Termination points are marked by a filled circle for Keplerian configurations and by an open circle for the instability with respect to axisymmetric perturbation. For the $2.1 M_{\odot}$ curve, notice a tiny thick segment, corresponding to the relativistic spin up by the angular momentum loss, just before the axisymmetric instability limit is reached. Note the change of sign of $\mathrm{d} f / \mathrm{d} J$ for the dashed (isolated pulsars) curves, which signals the end point of the back-bending stage, denoted by thick dashed line segments. There is no back-bending for accreting stars of similar baryon masses, for which $\mathrm{d} f / \mathrm{d} J$ stays positive along all evolutionary track. For comparison we plotted one curve with $x_{l}=0.75$ (dotted line).

\section{Conclusion}

We studied the spin-up of accreting NSs with hyperonic cores and, in particular, the impact of the hyperon softening of the EOS of dense matter on the spin-up history. The spin-up sequences ended either at the Keplerian (mass shedding) limit or, for sufficiently high initial baryon mass, by an instability with respect to axi-symmetric perturbations leading to collapse into a Kerr black-hole. We considered an EOS that exhibits a strong back-bending behavior in the spin-down of isolated NSs. However, this EOS does not show back-bending in the spin-up by accretion, even in the most efficient spinning-up, without any angular momentum loss in the accretion process. Therefore, for hyperonic EOSs back-bending in the pulsar, timing does not imply spin clustering in accreting NSs in low-mass $\mathrm{X}$-ray binaries (LMXBs). Generally, our calculations show that the additional increase in mass that accompanies increase in angular momentum in accreting NSs weakens or even suppresses the back-bending phenomenon in the time evolution of NS rotation frequency. It should be stressed that this suppression of 
the BB phenomenon for hyperon EOSs does not mean that the effect cannot be theoretically obtained for very strong (perhaps unrealistically strong) softening of the EOS. In such a case, the back-bending present in the spin-down timing will also present be (albeit in a much weaker form) in the accretion spin-up, provided, of course, that not too large a fraction of the angular momentum is lost during the accretion process. This aspect of the impact of the high-density softening of the EOS on the spin evolution of neutron stars and, in particular, its observational signatures in the timing of isolated and accreting NSs will be the subject of our forthcoming publication.

Acknowledgements. This work was partially supported by the Polish MNiI Grant No. 1 P03D 00827 and by the CNRS/PAN LEA Astr-PF.

\section{References}

Afshordi, N., \& Paczynski, B. 2003, ApJ, 592, 354

Agol, E., \& Krolik, J. H. 2000, ApJ, 528, 161
Balberg, S., \& Gal, A. 1997, Nucl. Phys. A., 625, 435

Balberg, S., Lichtenstadt, I., \& Cook, G. P. 1999, ApJ, 121, 515

Cameron, A. G. W. 1959, ApJ, 129, 676

Glendenning, N. K., Pei, S., \& Weber, F. 1997, Phys. Rev. Lett., 79, 1603

Glendenning, N. K., \& Weber, F. 2001a, ApJ, 559, L119

Glendenning, N. K., \& Weber, F. 2001b, in Physics of Neutron Star Interiors, ed. D. Blaschke, N. K. Glendenning, \& A. Sedrakian, Lecture Notes Phys., 578, 305

Glendenning, N. K., \& Weber, F. 2002, AIP Conf. Proc., 610, 470

Nozawa, T., Stergioulas, N., Gourgoulhon, E., \& Eriguchi, Y. 1998, A\&A, 139, 431

Poghosyan, G., Grigorian, H., \& Blaschke, D. 2001, ApJ, 551, L73

Spyrou, N. K., \& Stergioulas, N. 2002, A\&A, 395, 151

Zdunik, J. L. 2000, A\&A, 359, 311

Zdunik, J. L., Haensel, P., \& Gourgoulhon, E. 2002, A\&A, 381, 933

Zdunik, J. L., Haensel, P., Gourgoulhon, E., \& Bejger, M. 2004, A\&A, 416, 1013 (Paper I) 